\title{
A SIP-based Method for Intra-Domain Handoffs
}

\author{
Dimitra Vali \\ OTE Research \\ Hellenic Telecommunications Organization - OTE S.A. \\ Athens, Greece \\ dvali@oteresearch.gr
}

\begin{abstract}
The mobility management issue in IP access networks can be dealt with from various perspectives. A possible approach includes the use of the Session Initiation Protocol (SIP) and its associate entities for mobility signaling. Following this approach, existing SIP functionality in a network can be reused for mobility management purposes, providing efficient utilization of resources. In this paper we propose the Hierarchical Mobile SIP (HMSIP) framework, a SIP-based intra-domain mobility management approach that builds upon the general framework of SIP mobility management. The HMSIP Agent is introduced as a border network entity providing the necessary mobility functionality. Detailed location and mobility management procedures are presented. The scheme is further enhanced with additional functionality for the support of multiple HMSIP Agents across the border of a domain, maintaining a unique contact address throughout a session within the domain.
\end{abstract}

Keywords: SIP, handoff, micro-mobility.

\section{INTRODUCTION}

IP networks tend to become the ubiquitous infrastructure for every telecommunication exchange. One of the most important challenges IP networks face is mobility support. Mobile IP (MIP) [1] and Mobile IPv6 (MIPv6) [2] are the results of IETF mobility support efforts. The Mobile IP protocols enhance the network (IP) layer, so that IP hosts can change location and retain their communicating sessions. In addition, various micro-mobility approaches (e.g. Cellular IP [3], HAWAII [4], HMIPv6 [5], IDMP [6]) have also been developed for enhancing the Mobile IP performance in high mobility environments. Micro-mobility schemes enable fast intra-domain handoff by constraining mobility related signaling inside the boundaries of the domain.

Mobility in IP networks can be alternatively supported by application layer mobility protocols that rely on higher layer signaling to achieve the sought results. Some of these efforts include the use of the Session Initiation Protocol (SIP) [7]. For consistency reasons, we will refer to those schemes as "Mobile SIP (MSIP)" (in accordance to "Mobile IP (MIP)"). Motivation and description of Mobile SIP functionality can be found in [8], [9] and [10]. According to these proposals, SIP can be used to provide terminal mobility to Internet multimedia applications, with the appropriate SIP extensions to the base SIP specification. Specifically, SIP signaling is used after the handoff for the end-to-end session re-establishment of SIP ongoing sessions between the communicating users. The newly acquired IP address of the mobile host is communicated to the corresponding host via SIP methods and subsequent data are sent to the new location of the user.

\author{
Sarantis Paskalis, Alexandros Kaloxylos, Lazaros Merakos \\ Department of Informatics and Telecommunications \\ University of Athens \\ Athens, Greece \\ \{paskalis, agk, merakos\}@di.uoa.gr
}

The major argument for using Mobile SIP to achieve terminal mobility in SIP environments is the reuse of existing SIP infrastructure (i.e. SIP proxies, SIP registrars, etc.) for the functionality required by mobile hosts. The deployment of Mobile IP leads to some extent in a duplicated network functionality and stored user data. Both SIP and Mobile IP have their own mechanism for registration/location update and their own database for storing location info. Additionally, the use of Mobile SIP could also compensate for the current lack of wide deployment of Mobile IP.

However, Mobile SIP needs to be optimized for fast handoffs in order to handle mobility in IP networks efficiently. Without optimization, the communication re-establishment after a handoff requires end-to-end signaling exchange that results in long update delays and high signaling overhead in the backbone network. This is especially critical in cases where mobility of resource reserving QoS flows is entailed. The mobility related end-to-end signaling exchange results in endto-end reservation re-establishment, long handoff delay and bandwidth waste through over-reservation. Therefore, micromobility enhancements are also necessary in SIP environments (similar to Mobile IP environments) to handle intra-domain handoff efficiently. Mobile IP-based micro-mobility schemes if applied will also lead to duplication of functionality in the deployed networks. Hence, enhancing the Mobile SIP approach to cater for micro-mobility seems to be the most efficient solution for enabling fast handoff in SIP networks. An RTP [11] translator optimization for Mobile SIP is proposed in [9] to accomplish this task, which caters, however, only for RTP flows and induces several modifications to the standard RTP discard behavior of the mobile terminal.

In this paper, we introduce a SIP-based micro-mobility scheme, named Hierarchical Mobile SIP (HMSIP) that seeks to efficiently handle micro-mobility in SIP environments. Our approach assumes the existence of one or more SIP-based domain border entities (named HMSIP Agents), which are responsible for handling the intra-domain mobility locally. HMSIP Agent functionality may be centralized or distributed across numerous border entities. Our work specifically focuses on the specification of efficient mobility procedures that enable seamless mobile host handoff between HMSIP Agents belonging to the same domain. The procedures described are also sufficient for the treatment of the centralized HMSIP Agent case. In our proposed scheme, SIP signaling is the preferred means of micro-mobility signaling. If the corresponding host does not support SIP, the sessions will not be disrupted, when the mobile host moves within its roaming domain. If the corresponding host supports SIP, it will also be 
able to locate the mobile host even if it changes places of attachment. Since SIP is only used for intra-domain mobility signaling, all types of traffic can be accommodated.

The remainder of this paper is organized as follows: Section II outlines the architecture of the HMSIP framework, and presents the main entities of our proposal. Section III expands on the functionality of the framework and presents the necessary signaling message flows and state maintenance for the registration and handoff procedures. Finally, section IV concludes the paper and hints towards future work.

\section{HMSIP ARCHITECTURE}

HMSIP aims at reducing handoff latency and minimizing signaling overhead in the backbone network, by restricting intra-domain handoff related signaling inside the roaming domain. All types of IP traffic are handled by HMSIP, including TCP flows.

HMSIP relies on Mobile SIP functionality for inter-domain mobility, much like the various network layer micro-mobility schemes rely on Mobile IP for global roaming. Our proposal follows the general regional registration approach found in various proxy-Agent micro-mobility schemes (e.g. MIP-RR [12], HMIPv6 [5], IDMP[6]) and builds on the SIP Hierarchical Registration proposed in [9].

A key entity in HMSIP architecture is the HMSIP Agent. It is a SIP Mobility Agent that is located at the domain border. The HMSIP Agent contains the necessary intelligence for localizing the intra-domain mobility related signaling and performing fast data path redirection to the current location of the mobile. Its functionality may be distributed across various domain border entities, as it is shown in Figure 1.

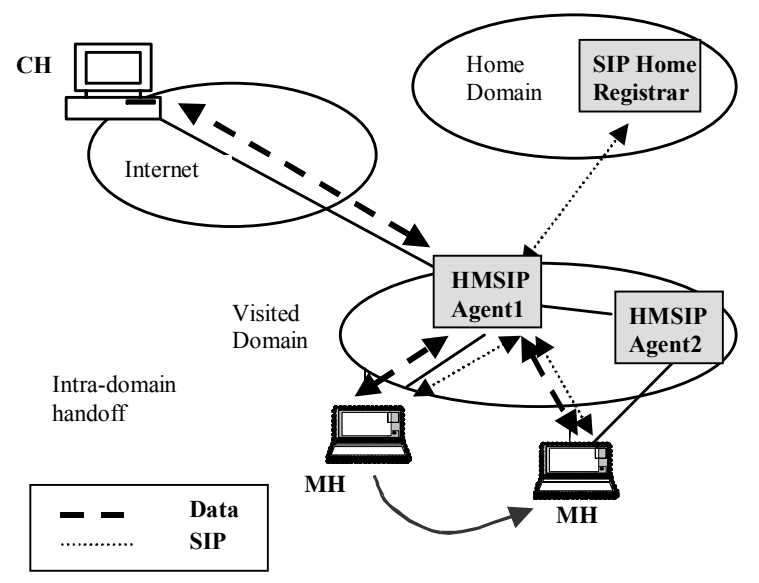

Figure 1 HMSIP network reference architecture

Similarly to other micro-mobility approaches, HMSIP allocates two IP addresses to a mobile host $(\mathrm{MH})$ entering a visited domain, a Local Address (LA) and a global Domain Address (DA). The LA is an IP address that reflects the current point of attachment of the $\mathrm{MH}$ and is routable inside the visiting domain. It may even be a private address inside the domain. It is allocated to the $\mathrm{MH}$ by the serving access router. After a handoff to a new access router, the $\mathrm{MH}$ always gets a new LA. The DA is a globally routable IP address assigned to a $\mathrm{MH}$ that does not change as long the $\mathrm{MH}$ roams inside an access domain and has active sessions. The DA is allocated to the $\mathrm{MH}$ by the serving HMSIP Agent, drawing it from a pool of public IP addresses. The global IP address assigned to the mobile host remains unmodified throughout its active communication inside the roaming domain. The existence of a stable DA further allows for smooth interworking with nonmobility aware protocols such as QoS enabling RSVP [13].

The building blocks of the HMSIP Agent are shown in Figure 2. It is essentially a domain border router, enhanced with the functionality of a SIP Proxy/B2BUA and a SIP Registrar. The HMSIP Agent is responsible for maintaining and managing a database with soft state mappings between the SIP URI, the DA and the LA for the mobiles roaming inside the domain. Soft states allow for resilience and error recovery in case of unexpected events. The HMSIP Agent releases allocated addresses to a $\mathrm{MH}$ when the $\mathrm{MH}$ stops communicating with the HMSIP Agent for any reason (out of domain boundaries, crashed, etc.)

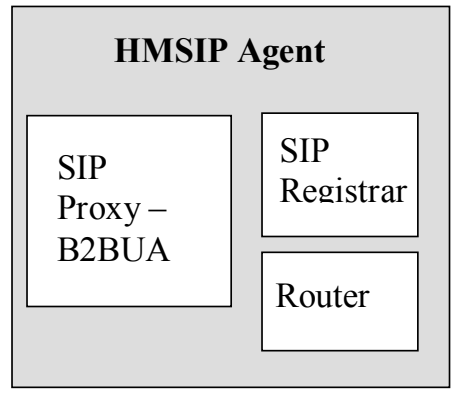

Figure 2 HMSIP Agent architecture

\section{HMSIP FUNCTIONALITY}

To give an overview of the HMSIP functionality, we follow the simple example illustrated in Figure 1. We assume that a $\mathrm{MH}$ powers-on inside (or enters) a visited domain, where it is allocated an LA (LA1) from the serving access router (AR), as well as a global DA (DA1) from the serving HMSIP Agent1. The DA1 is registered with the home Registrar of the $\mathrm{MH}$, while the LA1 is registered with the serving HMSIP Agent1.

We then assume that the $\mathrm{MH}$ establishes a session (SIP, TCP or any other) with a corresponding host $(\mathrm{CH})$. All subsequent signaling and data sent towards the $\mathrm{MH}$ from the exterior of the domain use the DA1 as the MH address. Since DA1 is allocated by and associated with HMSIP Agent1, all packets sent towards the MH arrive at HMSIP Agent1. HMSIP Agent1 tunnels all packets destined to DA1 towards LA1.

During its ongoing session, we assume that the MH performs a handoff to an access router controlled by HMSIP Agent2. The $\mathrm{MH}$ is then allocated a new local address LA2 from the new serving AR. If the $\mathrm{MH}$ has active sessions, it retains its previously acquired DA1 and keeps communicating through HMSIP Agent1, until all active sessions terminate. If the $\mathrm{MH}$ has no active sessions, as well as when its active sessions terminate, it re-acquires a pair of DA, LA and acts as 
newly powered on device in the HMSIP Agent2 controlled area.

The serving HMSIP Agent is being kept informed about the current location (LA) of the MH inside the domain via the SIP REGISTER method. It is, therefore, able to properly redirect any flows to the new MH location. The method described eliminates the need for (SIP or other) end-to-end session reestablishment following an intra-domain handoff.

In what follows, we describe the HMSIP signaling exchange and state modifications that take place in the cases of power-on and intra-domain handoff in detail. The inter-domain handoff case is dealt by normal Mobile SIP functionality and is not further discussed in this paper.

\section{A. Power-on procedures}

When powering-on inside (or entering) a visiting domain, the $\mathrm{MH}$ is allocated a LA (LA1) from the serving access router, as well as a DA (DA1) from the serving HMSIP Agent1. The new LA and DA should be registered with the MH's visited (Regional Registration) and home (Home Registration) network respectively, via the respective SIP registration procedures.

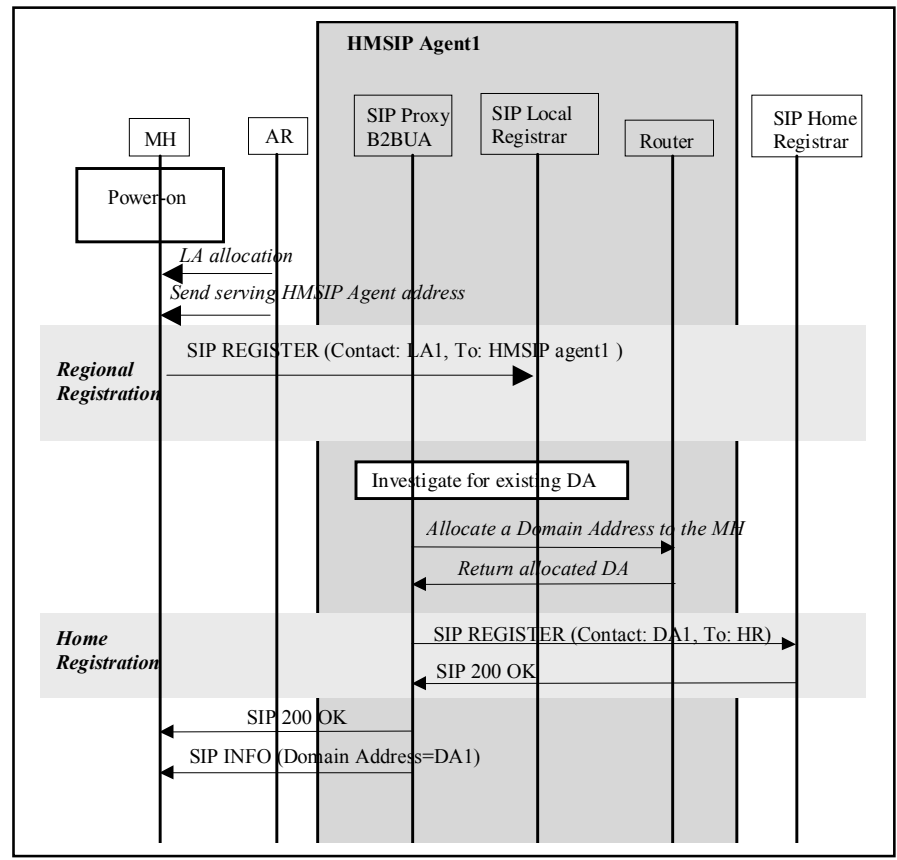

Figure 3 HMSIP Regional and Home Registration

The signaling exchange occurring during the registration procedure is depicted in Figure 3. After receiving a local address (LA1) from the local access router, the MH sends a SIP REGISTER message towards its serving HMSIP Agent1 to register its new location with the visited network (Regional Registration). The address of the serving HMSIP Agent 1 is advertised to the $\mathrm{MH}$ by its serving access router. The Contact field of the REGISTER message includes the LA1. The SIP Registrar entity of the HMSIP Agent, after receiving this REGISTER message, queries its database about the existence of the specific SIP URI and its association with any LA and
DA. If a relative record is found, then the LA stored is checked. If the LA1 received is the same as the LA found in the database, the record is refreshed. If the LA1 received is different than the LA stored, then the record is updated to reflect the current position of the $\mathrm{MH}$. In both cases the registration procedure stops there (Regional Registration).

In the event of no record found containing the sought SIP URI, the MH is considered as a new arrival to the HMSIP agent1. The HMSIP Agent1 allocates a DA (DA1) to the MH, and stores the new record of mappings between the SIP URI, the DA1 and the LA1 to its Registrar entity. This record is refreshed by periodic REGISTER messages sent by the MH.

When the DA is allocated and the local registration procedures are complete, the SIP Local Registrar instructs the SIP proxy/B2BUA entity of the serving HMSIP Agent to trigger the respective Home Registration procedure for registering DA1 with the home network of the MH. HMSIP Agent initiates a REGISTER message towards the SIP Home Registrar of the $\mathrm{MH}$, whose location can be retrieved from the SIP URI of the MH. The REGISTER message contains DA1 in the Contact field. As a result, the SIP Home Registrar creates a mapping between the SIP URI and the DA of the MH in its database. SIP calls towards the $\mathrm{MH}$ can now be routed to the DA1 (Figure 4).

Finally, the HMSIP Agent1 communicates the allocated DA1 to the MH, including it inside the payload of a SIP INFO message. The DA will be subsequently used as the static address of the $\mathrm{MH}$ inside the roaming domain. The $\mathrm{MH}$ uses the DA as the source address of its outgoing packets before tunneling them to HMSIP Agent1, as shown in Figure 4 (the encapsulation header contains LA1 as source address for topology correctness.) In the reverse direction, the packets destined for the MH will be sent to DA1, and the HMSIP Agent1 will tunnel them towards LA1 inside the domain.

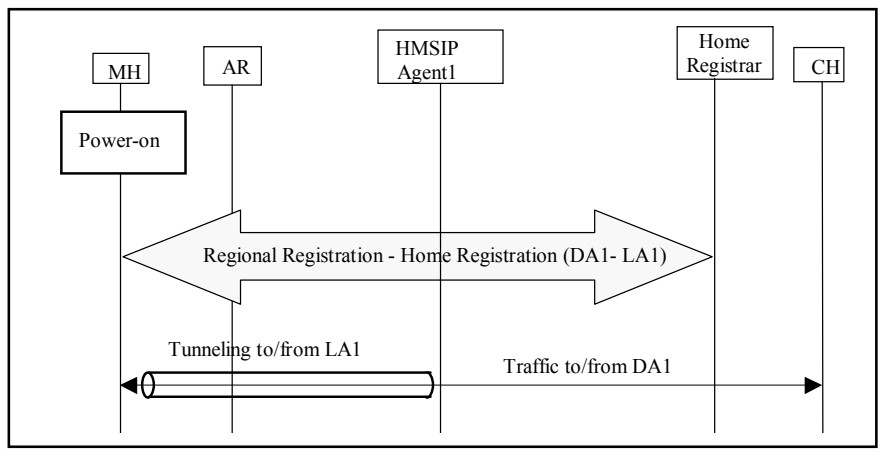

Figure 4 Session establishment and data exchange following power-on

The domain gateway for all traffic to and from the MH is the serving HMSIP Agent1. As it will be described in the next sections, the serving/gateway HMSIP Agent will not change for as long as the $\mathrm{MH}$ roams inside the domain during an active communication. The MH stores the advertised HMSIP Agent1 address as the address of the Gateway HMSIP Agent for future reference. 


\section{B. Handoff procedures}

The goal of our micro-mobility approach is the maintenance of the same global IP address DA by the mobile for as long as it roams inside the same domain during active sessions. In a reference network, such as the one in Figure 1, handoffs may occur, in which the $\mathrm{MH}$ attaches to an access router (AR) controlled by the same or a different HMSIP Agent. The fulfillment of the micro-mobility requirement suggests that the serving HMSIP Agent for a MH remains the same for as long as the $\mathrm{MH}$ has active sessions and roams inside the domain.

To facilitate the description of our scheme, we assume that the following states characterize the roaming behavior of a $\mathrm{MH}$ (Figure 5):

- $\quad$ Locally Served (LS)

- $\quad$ Remotely Served (RS)

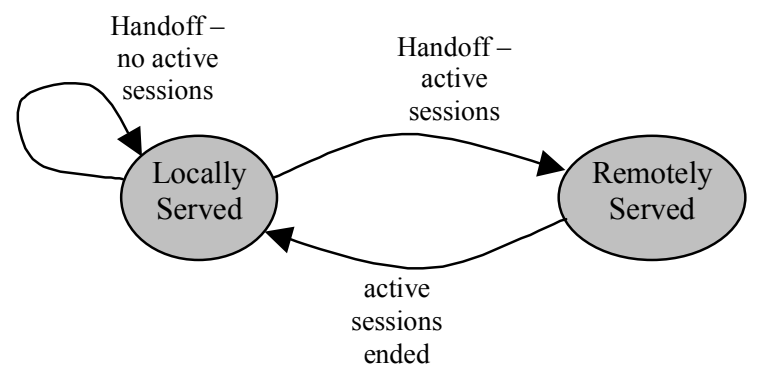

Figure 5 State transition in handoffs

A MH is at state LS when its serving HMSIP Agent is the local HMSIP Agent controlling its current AR. A MH is at state RS when its serving HMSIP Agent is not the local HMSIP Agent. The mobile enters state LS, when it powers on and receives a LA and DA from its local HMSIP Agent. Both LS and RS are soft states, refreshed periodically by REGISTER messages sent by the MH. Additionally, there may be an upper limit to the amount of time a $\mathrm{MH}$ is in the RS state continuously, to force topology restructuring.

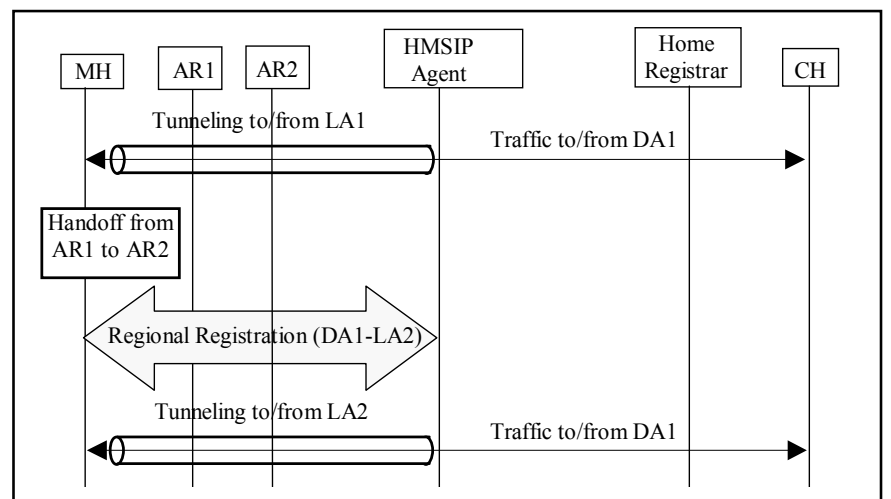

Figure 6 Intra-HMSIP Agent handoff

When the $\mathrm{MH}$ hands off to a different access router, it checks whether it has changed HMSIP Agent. The MH can check this by comparing the advertised HMSIP Agent address with its internally stored gateway HMSIP Agent address. In case of an intra-HMSIP Agent handoff (same local and gateway agents), the $\mathrm{MH}$ remains at state LS. A new LA2 is allocated from the access router, while the DA1 remains the same. The Regional Registration procedure is triggered (Figure 6), resulting in updating the DA-LA mapping in the HMSIP Agent to reflect the new LA.

If the $\mathrm{MH}$ performs an inter-HMSIP Agent handoff and has no active sessions, the $\mathrm{MH}$ acts as in the initial power-on case, re-entering the LS state in the new environment. The $\mathrm{MH}$ acquires a new LA2 as well as a new DA2 from the local HMSIP Agent2, which becomes the serving HMSIP Agent for the MH. No connection with the old serving HMSIP Agent1 is necessary, since there are no active sessions that need to be continued. The MH re-initiates both Home and Regional Registration procedures towards its new serving HMSIP Agent, as depicted in Figure 3.

If, however, the MH hands off to HMSIP Agent2 while in the middle of an active data communication, it switches to the RS state. The MH is allocated a local address LA2 but not a new DA. The MH initiates only the Regional Registration procedure towards its previously active HMSIP Agent1 (Figure 7). All signaling and data transport take place between the $\mathrm{MH}$ and the old gateway HMSIP Agent1. After a successful registration process, the $\mathrm{MH}$ is entering the RS state both in its internal state machine and in the state mappings in the database of HMSIP Agent1. HMSIP Agent1 is refreshing the RS state (and DAs) for the respective MHs through periodic REGISTER messages from the MHs. The HMSIP Agent may impose a time limit on mobiles in state RS, after which, it would remove their entries from its database. HMSIP Agent2 does not participate in any mobility-related SIP signaling or data encapsulation/decapsulation for the $\mathrm{MH}$.

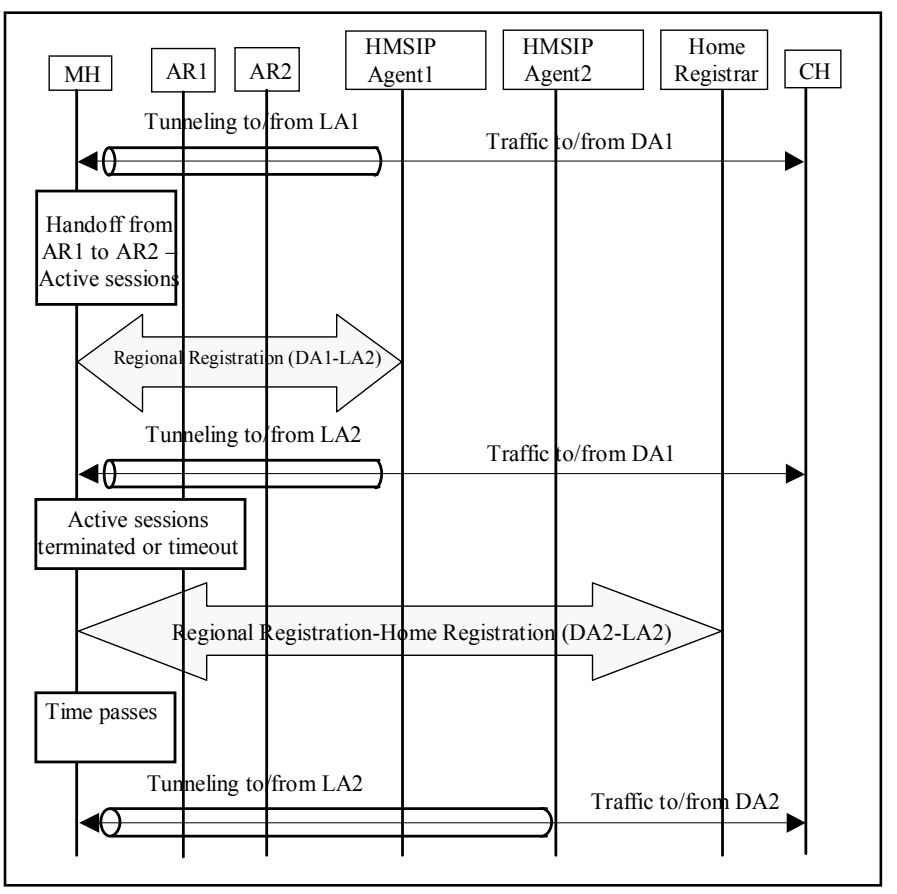

Figure 7 Inter-HMSIP Agent Handoff with active sessions. 
When all active sessions of the $\mathrm{MH}$ are terminated or the maximum time limit in RS state is reached, the $\mathrm{MH}$ acts as in the inter-HMSIP Agent handoff without active connections. It re-initiates the full registration procedure with HMSIP Agent2, acquiring a new DA (DA2) and entering state LS in its new environment (Figure 7). Alternatively, HMSIP Agent1 will receive no refresh messages any more from the $\mathrm{MH}$ for refreshing DA1, thus, HMSIP Agent1 will release DA1 after the refresh timeout expiration.

\section{CONCLUSIONS}

In this paper, we presented the Hierarchical Mobile SIP (HMSIP) framework, an efficient micro-mobility management scheme for SIP networks. The location and mobility management procedures were described. HMSIP minimizes intra-domain handoff latency by restricting mobility related signaling inside the roaming domain, while taking advantage of information stored in existing SIP network entities. Moreover, HMSIP supports intra-domain mobility for all types of traffic and sets no specific requirements to the far end-system.

To locally handle intra-domain mobility, HMSIP introduces a SIP-based domain border entity, named HMSIP Agent. The HMSIP Agent terminates the handoff related signaling inside the domain and performs fast data path redirection to the current location of the mobile.

HMSIP is further enhanced by redundancy and scalability features, supporting multiple HMSIP Agents at the network border. Intra-domain handoff to a different serving HMSIP Agent is specially treated and the respective HMSIP mobility procedures are described in detail. Message flow diagrams are given describing the mobile host registration procedure and the proper re-direction of the data path to the current location of the mobile host after intra-domain handoff.

Future work will comprise the development in a simulation environment of the HMSIP intra-domain handoff procedures. The proposed scheme's efficiency will be validated via comparison with Mobile SIP and other micro-mobility approaches.

\section{REFERENCES}

[1] C. Perkins ed., "IP Mobility Support for IPv4", RFC 3344, August 2002.

[2] D. Johnson, C. Perkins, J. Arkko, "Mobility Support in IPv6", Internet Draft (Work in progress), June 2003.

[3] A. Campbell, J. Gomez, S. Kim, A. Valko, Wan Chien-Yin, Z. Turanyi, "Design, Implementation, and Evaluation of Cellular IP", IEEE Personal Communications, Vol. 7, pp. 42-49, August 2000.

[4] R. Ramjee, T. La Porta, S. Thuel, K. Varadhan, S. Y. Wang, "HAWAII: a Domain-Based Approach for Supporting Mobility in Wide-Area Wireless Networks", Proceedings of the Seventh International Conference on Network Protocols (ICNP) 1999, pp. 283-292.

[5] H. Soliman, C. Castelluccia, K. El-Malki, L. Bellier, "Hierarchical IPv6 Mobility Management (HMIPv6)", Internet Draft (work in progress), June 2003.

[6] A. Misra, S. Das, A. Datta and S. K. Das, "IDMP-based Fast Handoffs and Paging in IP-Based 4G Mobile Networks", IEEE Communications Magazine, vol. 40, no. 3, pp. 138-145, March 2002.

[7] J. Rosenberg, H. Schulzrinne, G. Camarillo, A. Johnston, J. Peterson, R. Sparks, M. Handley, E. Schooler, "SIP: Session Initiation Protocol", RFC 3261, June 2002.

[8] E. Wedlund, H. Schulzrinne, "Mobility support using SIP", 2nd ACM/IEEE International Conference on Wireless and Mobile Multimedia, Seattle, Washington, Aug. 1999

[9] H. Schulzrinne, E. Wedlund, "Application layer mobility using SIP", Mobile Computing and Communications Review, Volume 4, Number 3

[10] A. Dutta, F. Vakil, J.-C. Chen, M. Tauil, S. Baba, N. Nakajima, and H. Schulzrinne, "Application layer mobility management scheme for wireless Internet", In Proc. of IEEE International Conference on Third Generation Wireless and Beyond (3Gwireless'01), (San Francisco, CA), pp. 379-385, May 2001.

[11] H. Schulzrinne, S. Casner, R. Frederic, V. Jacobson, "RTP: A transport protocol for real-time applications", IETF RFC 1889, Jan. 1996

[12] E. Gustafsson, A. Jonsson, C. Perkins, "Mobile IP Regional Registration", Internet Draft (work in progress), March 2001

[13] R. Braden ed., L. Zhang, S. Berson, S. Herzog, S. Jamin, "Resource ReSeRvation Protocol - Version 1 Functional Specification”, RFC 2205, September 1997. 